\title{
Our Experience with Carotid Body Paragangliomas
}

\section{Erdogan B. A. ${ }^{1}$, Bora F. ${ }^{2}$, Altin G. ${ }^{1}$, Paksoy M. ${ }^{1}$}

${ }^{1}$ Department of Otolaryngology, Dr. Lutfi Kirdar Kartal Education and Research Hospital, Istanbul, Turkey;

2Department of Otolaryngology, Kafkas University, Kars, Turkey

Received March 12, 2012; Accepted October 10, 2012.

Key words: Carotid body paraganglioma - Shamblin classification - Treatment

Abstract: Carotid body paragangliomas (CBP) are rare neoplasms arising from the small chemoreceptor organ in the adventitia of the common carotid bifurcation. The aim of this study is to present the diagnostic process, performed treatment and obtained results in patients with carotid body paragangliomas of the Department of Otolaryngology of Istanbul Education and Research Hospital between March 1997 and November 2008. Retrospective analysis was carried out, based on the medical documentation of 5 patients with carotid body paragangliomas ( 3 women and 2 men), age range 44 to 68 years with a mean of 59.6 years. Four of the patients were diagnosed and treated with Shamblin type II tumor, one of the patients with type I. Physical examination, radiological evaluation, method of the treatment and post-treatment complications were studied. The most common and single symptom was nonspecific neck mass. Preoperative diagnostic evaluation consisted of a color duplex ultrasonography, computerized tomography with contrast enhancement, magnetic resonance imaging and digital subtraction angiography. In all patients with Shamblin type I and II, blunt dissection of the tumor was conducted smoothly in the subadventitial plane. Postoperative vagus nerve and hypoglossal nerve deficit were reported in one case. Carotid body paraganglioma excision has higher risk of cranial nerve paresis and carotid artery injury, so it requires careful handling and good surgical skills to ensure complete removal.

Mailing Address: Banu Atalay Erdogan, MD., Department of Otolaryngology, Dr. Lutfi Kirdar Kartal Education and Research Hospital, 34731 Istanbul, Turkey; Phone: +902 164413 900; Fax: +902 163520 083; e-mail: banuatalay81@gmail.com 


\section{Introduction}

Paragangliomas are neuroendocrine tumors derived from paraganglia of the parasympathetic nervous system. Paraganglia play an important role in homeostasis of the organism by acting directly as chemoreceptors or by the secretion of catecholamines in response to stress (Baysal, 2002). Paragangliomas of the head and neck (HNPs) are rare, comprising about $0.6 \%$ of head and neck tumors and about $0.03 \%$ of all tumors (Lee et al., 2002). They most commonly (60\%) occur at the carotid bifurcation. Additional sites of origin frequently include the jugular bulb (JP), the vagal nerve (VP) at the nodose ganglion, the middle ear (tympanic paraganglioma) (Pellitteri et al., 2004). Carotid body paragangliomas are most commonly unilateral and occur in a sporadic pattern. Multiple paragangliomas account for $11-22 \%$ of all cases. The $10-50 \%$ of paragangliomas are familial (Bikhazi et al., 1999). Classic tumor syndromes associated with a high incidence of paragangliomas include multiple endocrine neoplasia type II (MEN II), von Hippel-Lindau disease and neurofibromatosis type I (NF I) (Neumann et al., 2002). Specific genetic abnormalities are associated with paragangliomas such as deletions at chromosomes 11q13 and 11q22-23 where the tumor suppressor gene PGL1 has been located (Bikhazi et al., 2000). Paragangliomas are benign neoplasms in most cases; overall less than $10 \%$ of all paragangliomas have been cited to be malignant (Lee et al., 2002). Physical examination of CBP reveals a nontender, rubbery, pulsatile lesion that is more easily moved laterally than vertically (Fontaine's sign). Due to the highly vascular nature of paragangliomas, an open or closed biopsy should not be attempted (Wang et al., 2000). In order to establish the diagnosis of a HNP, imaging modalities such as ultrasonography, computerized tomography (CT), magnetic resonance imaging (MRI) and digital subtraction angiography (DSA) are important (Stoeckli et al., 2002). Shamblin et al. classified the carotid body tumors according to carotid artery involvement in 1971. In type I the carotid artery is minimally involved with the tumor. In type II, the tumor partially encases the carotid arterial structures. Type III is the most invasive of arterial structures (Shamblin et al., 1971). The different therapeutic options include surgical extirpation, conventional radiotherapy and stereotactic radiosurgery (Foote et al., 2002). In selected cases a "wait and scan" policy or permanent embolization may be discussed (Persky et al., 2002). When tumor excision can be complete and do not cause disability resulting from compromise of function of multiple cranial nerves, surgery is the key treatment. Early surgical intervention should be considered in patients who are deemed satisfactory surgical candidates (Marchesi et al., 1999).

\section{Material and Methods}

A retrospective review of the medical records of the patients diagnosed with carotid bifurcation paragangliomas during the period from March 1997 and November 2008 was performed. Surgical and therapeutic data were collected from reviewing of the medical and surgical records of the 5 operated carotid 
bifurcation paragangliomas. Four of the patients were diagnosed and treated with Shamblin type II tumor, one of the patients with type I. The main symptom patients exhibited was a slowly growing mass on the neck. None patients did not have any neurological squeal. On examination there was a pulsatile, hard and rubbery mass located below the mandibular angle which was palpable on the carotid arterial trace, mobile on the horizontal plane and immobile on the vertical plane. All surgical patients were operated on by an experienced head and neck senior surgeon. Preoperative diagnostic evaluation consisted of a color duplex ultrasonography, computerized tomography with contrast enhancement and magnetic resonance imaging. Digital subtraction angiography through femoral artery catheterization followed the initial evaluation and proved diagnostic in some cases. Microcatheter embolization after visualization of vessels feeding the tumor was performed using straight and curled microcoils (Microcoils, William Cook Europe, Denmark) as the main embolic material together with Gelfoam and polyvinyl alcohol (PVA) in four out of five carotid body tumors (Figure 1). Surgery was performed within 48 hours of embolization to avoid development of collateral vessels for tumor blood supply and the initiation of postembolization inflammatory effect. Surgical excision was performed under general anaesthesia. Common carotid artery and branches
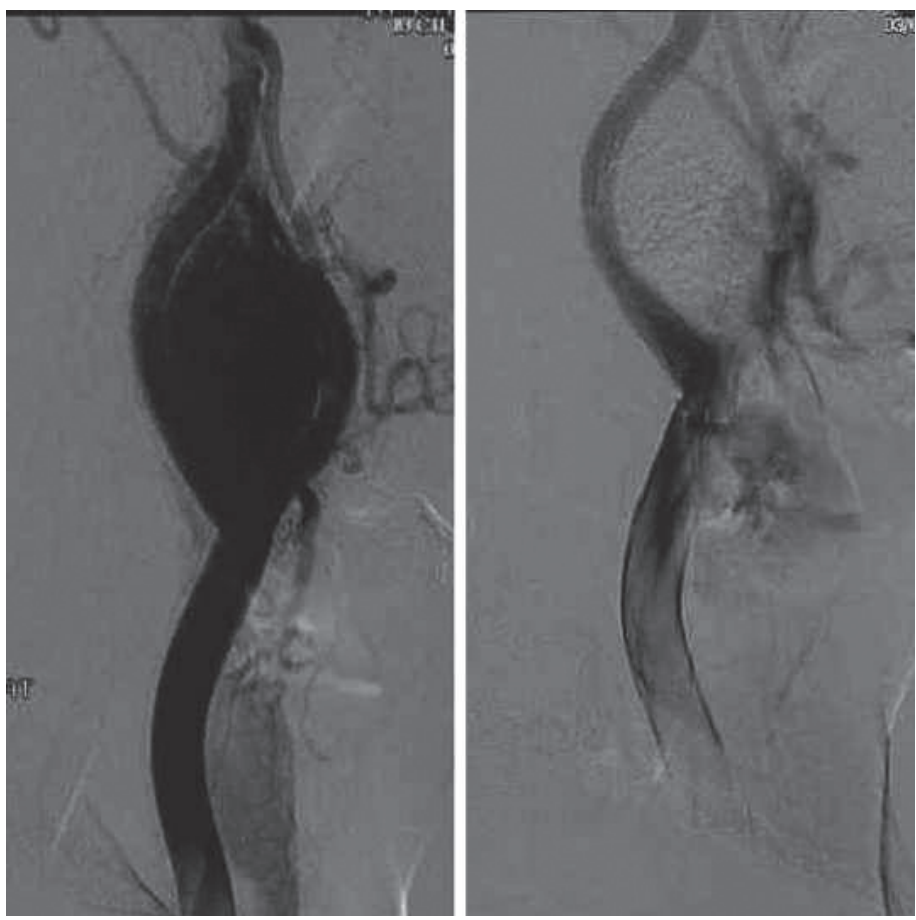

Figure 1 - DSA imaging of a right CBP and the same tumor after selective microcoil embolization. Significant reduction in vascular blush indicates reduced arterial supply to the tumor. 
were explored. Vagus and hypoglossal nerves were dissected and separated from the surgical plane in order to prevent damage. The tumor was dissected completely from the common carotid artery and its branches in the subadventitial plane. All resected paragangliomas were examined under light microscopy for the characteristic arrangement of the tumor cells in distinctive cell balls, which is called Zellballen. Immunohistochemistry analysis was performed using specific antibodies against chromogranin, synaptophysin, neuron-specific enolase, serotonin, and S-100 protein. The median postoperative hospital stay was 5 days.

\section{Results}

The ages of patients ranged from 44 to 68 years with a mean of 59.6 years. Three of our patients were female and two of patients were male. All patients had no familial history of paraganglioma and presented with multiple head and neck paraganglioma. The most common presenting symptom was a painless lateral neck mass. The mean period between onset of symptoms and presentation was 9 months with a range from 3 to 12 months (Table 1). No patient complained of symptoms related to a functional tumor with catecholamine hypersecretion such as palpitations, hypertension, tachycardia, or tremor. Four of CBPs $(80 \%)$ were grouped into class II and one (20\%) in class I. The dissection was performed in the relatively avascular subadventitial plane between the arteries. In one patient with Shamblin II external carotid artery was excised together with the mass. The mean greatest dimension at presentation was $2.1 \mathrm{~cm}$ (range 1.1-3.5 cm). No transient ischemic attack or stroke was noticed but; a female patient with Shamblin II developed post-operative vocal cord and hypoglossal nerve paralysis on the lesion side. Six months of follow-up found no remission in vocal cord paralysis. All masses were confirmed pathologically as paraganglioma and no trace of malignancy was found. Patients underwent control once in 6 months in the first postoperative year and once a year in the following years. No evidence of local or distant metastasis was seen at the follow-up checks.

\section{Table 1 - Age, mean duration of the symptoms, classification of the tumors and embolization of the paragangliomas}

\begin{tabular}{lcclc}
\hline Name & Age & $\begin{array}{c}\text { Mean duration } \\
\text { of symptoms (months) }\end{array}$ & Classification & Embolization \\
\hline C.A. & 62 & 12 & Shamblin II & $(+)$ \\
G.K. & 44 & 3 & Shamblin II & $(+)$ \\
A.K. & 68 & 12 & Shamblin II & $(+)$ \\
G.D. & 62 & 12 & Shamblin II & $(+)$ \\
N.K. & 62 & 6 & Shamblin I & $(-)$ \\
\hline
\end{tabular}




\section{Discussion}

Paragangliomas of the head and neck are rare, they most commonly (60\%) occur at the carotid bifurcation. CBPs often presents as a painless, slowly enlarging mass in the lateral neck (Patetsios et al., 2002). Differential diagnosis is very important because a variety of lesions can have the same clinical appearance such as lymphomas, branchial cleft cysts, parotid gland tumors, metastatic cervical lymphadenopathy, thyroid masses, and tuberculosis. CBPs typically present as slowly growing lateral neck masses. The lesion is more freely moveable horizontally than vertically because of adherence to the carotid artery (Patetsios et al., 2002). The finding of a carotid bruit or a pulsatile character of the tumor supports the diagnosis of CBP. Evidence of increased endocrine activity demonstrates only $1-3 \%$ of CBP. In our study, the presenting symptoms of CBPs were neck mass and there was no patient with clinical signs of a hyperfunctioning paraganglioma. The mean duration of symptoms is $17.44 \pm 18.68$ months. In case of a tumor with a suspected endocrine activity, laboratory studies (urine metanephrine, normetanephrine, and plasma catecholamines) should be obtained. A painless neck mass is also the most frequent symptom in patients with VPs (Miller et al., 2000). In our cases there is no vagal paraganglioma. Evaluation by an imaging procedure is absolutely necessary to establish the diagnosis of a CBP. Also imaging procedure is important for treatment planning (Stoeckli et al., 2002). B-mode sonography in combination with color-coded Doppler sonography is an inexpensive, non-invasive, readily available diagnostic tool that is frequently used as the first imaging step for cervical paragangliomas (Stoeckli et al., 2002). Stoeckli et al. (2002) reported on 18 CBPs that were evaluated with color-coded Doppler sonography. In our study, $4(80 \%)$ of patients with CBP were screened initially with US. Large VPs may also lead to a splaying of the carotid bifurcation and might therefore be misinterpreted as a CBP (Thabet and Kotob, 2001). CT will show an enhancing mass. Bone windows can reveal skull-base erosion and intracranial extension. To classify JPs and TPs, the extend of temporal bone destruction is important. The preoperative classification of these tumors is essential, since the operative approach will be chosen depending on the tumor stage. All patients with JP and TPs were admitted to our department with a CT scan as a first imaging study. The MRI plays an important role in the pretherapeutic evaluation of cervical paragangliomas as well as temporal paragangliomas (Thabet and Kotob, 2001). MRI is more sensitive than CT for lesions involving the skull base or extending within the cranial vault (Pellitteri et al., 2004). Paragangliomas usually show a hyperintense signal on T2-weighted images and a distinct contrast enhancement on T1-weighted images (Hoegerle et al., 2003).

The definitive imaging procedure to confirm the diagnosis of a head and neck paraganglioma represents the DSA (Patetsios et al., 2002; Stoeckli et al., 2002). Carotid DSA, through percutaneous femoral catheterization, remains an invaluable diagnostic procedure for defining the exact vascular anatomy of the neoplasm. It provides an arterial "map" of the major feeding and the collateral vasculature 
supplying the tumor (Kafie and Freischlag, 2001). Later phases of the arteriogram visualize the venous outflow. According to Persky et al. (2002) bilateral DSA should be performed in all patients with CBTs, VPs and JPs. In patients with TPs, a DSA is not necessary since the potential side effects of this procedure outscale the diagnostic value in those tumors (Persky et al., 2002). Embolization can be performed with DSA for preoperative devascularization of the tumor. Preoperative selective embolization of the tumor remains a matter of debate as major complications such as cerebrovascular accidents might occur (Urquhart et al., 1994; Tikkakoski et al., 1997). For some authors, embolization allows safer surgical excision of the paraganglioma without undue haemorrhage. It decreases duration of the hospital stay, and decreases risk of intraoperative hypoglossal nerve injury (Miller et al., 2000). Operation is recommended to be performed within the first $48 \mathrm{~h}$ to minimize revascularization edema and local inflammatory response (Kafie and Freischlag, 2001). In our series of patients, microcoil embolization was the main technique performed after superselective catheterization of the major feeding arteries in $80 \%$ of CBTs. Only one patient couldn't be applied embolization. In our series, no cerebral embolism and serious intraoperative bleeding were reported. The absence of postembolization neurological complications indicates the safety and efficacy of this technique in experienced hands. Today, most authors support the embolization of CBPs and VPs (Miller et al., 2000; Wang et al., 2000; Persky et al., 2002) but this item is still a matter of discussion in international otolaryngological literature (Murphy and Brackmann, 1989; Litle et al., 1996; Marchesi et al., 1999). Metabolic imaging procedures such as the somatostatin receptor scintigraphy are only available at special centres.

The current treatment options for HNPs include surgical resection, conventional radiation therapy, stereotactic radiosurgery, permanent embolization or a combination of those modalities (Foote et al., 2002). The goal of surgery is complete tumor removal (Foote et al., 2002; Persky et al., 2002). Cervical paragangliomas are usually resected via a transcervical approach. All carotid body tumors were excised via transcervical approach. Four of them were performed embolization and they were operated in 48 hours after embolization. No neurological deficit and carotid injury were reported.

CBPs are usually classified according to criteria set forth by Shamblin. Class I CBTs are localized tumors with minimal attachment to the carotid vessels and surgical resection is generally not difficult. Class II tumors partially surround the carotid vessels. Class III CBPs intimately surround the carotid vessels and surgical resection is challenging and often requires temporary interruption of the cerebral circulation (Shamblin et al., 1971). In our series four tumors (80\%) were grouped into class II and one (20\%) in class I. The risk of postoperative neurological damage and peroperative undue haemorrhage are higher in Shamblin class III tumors (Wang et al., 2000). Anand et al. (1995) reported on the postoperative outcome of 1181 CBP patients. In $21.8 \%$ of cases, a permanent postoperative cranial 
nerve deficit was noted. Sanli et al. (2012) reported early post-operative loss of consciousness and hemiplegia on the same side as the lesion in the two of the male patients with a type II tumor. One of them died on the fifth post-operative day. Vascular complications account for a total of 9-28\% with an estimated risk of stroke up to $11 \%$ (Hirsch et al., 1980). An experienced operating team in vascular reconstructive techniques is strongly recommended for reducing the risk of a major vascular injury, especially in Shamblin class III tumors.

Radiation therapy and stereotactic radiosurgery aim to achieve long-term tumor control (Foote et al., 2002; Persky et al., 2002). Permanent embolization may be able to slow down tumor growth and should only be considered in a palliative setting. The role of tumor irradiation in the management of paragangliomas remains controversial. Long-term tumor control rates of up to $96 \%$ have been reported (Hinerman et al., 2001). But, frequent side effects of irradiation include acute dermatitis, alopecia, external otitis, serous otitis media, altered taste and xerostomia (Foote et al., 2002). Serious complications such as radionecrosis of the temporal bone $(1.7 \%)$, brain necrosis $(0.84 \%)$ and radiation-induced malignant disease $(0.28 \%)$ have been reported (Foote et al., 2002). In addition, stereotactic radiosurgery represents a rather new treatment modality for jugulo-tympanic paragangliomas (Feigenberg et al., 2002; Foote et al., 2002). The principal indications for the use of radiotherapy as a primary treatment in our institution include larger tumors where after careful staging resection may result in significant morbidity, patients in poor health status with concomitant medical conditions, or as an adjunct to incompletely excised tumors with skull base or intracranial invasion. One advantage of radiation therapy is to avoid the morbidity of surgery while offering a high probability (96 to $100 \%$ ) of tumor control (Thabet and Kotob, 2001).

\section{Conclusion}

Carotid bifurcation paragangliomas are rare and slowly growing tumors. We support preoperative embolization in patients with CBP. Surgery is the treatment of choice for most patients with CBPs. Early treatment of patients with paraganglioma optimizes the outcomes. Whenever surgical extirpation is too risky, conventional radiotherapy and stereotactic radiosurgery may be considered as alternative treatment options.

\section{References}

Anand, V. K., Alemar, G. O., Sanders, T. S. (1995) Management of the internal carotid artery during carotid body tumour surgery. Laryngoscope 105, 231-235.

Baysal, B. E. (2002) Hereditary paraganglioma targets diverse paraganglia. J. Med. Genet. 39, 617-622.

Bikhazi, P. H., Roeder, E., Attaie, A., Lalwani, A. K. (1999) Familial paragangliomas: the emerging impact of molecular genetics on evaluation and management. Am. J. Otol. 20, 639-643.

Bikhazi, P. H., Messina, L., Mhatre, A. N., Goldstein, J. A., Lalwani, A. K. (2000) Molecular pathogenesis in sporadic head and neck paraganglioma. Laryngoscope 110,1346-1348. 
Feigenberg, S. J., Mendenhall, W. M., Hinerman, R. W., Amdur, R. J., Friedman, W. A., Antonelli, P. J. (2002) Radiosurgery for paraganglioma of the temporal bone. Head Neck 24, 384-389.

Foote, R. L., Pollock, B. E., Gorman, D. A., Schomberg, P. J., Stafford, S. L., Link, M. J., Kline, R. W., Strome, S. E., Kasperbauer, J. L., Olsen, K. D. (2002) Glomus jugulare tumor: tumor control and complications after stereotactic radiosurgery. Head Neck 24, 332-338, discussion 338-339.

Hinerman, R. W., Mendenhall, W. M., Amdur, R. J., Stringer, S. P., Antonelli, P. J., Cassisi, N. J. (2001) Definitive radiotherapy in the management of chemodectomas arising in the temporal bone, carotid body, and glomus vagale. Head Neck 23, 363-371.

Hirsch, J. H., Killien, C., Troupin, R. H. (1980) Bilateral carotid body tumours and cyanotic heart disease. AJR Am. J. Roentgenol. 134, 1073-1075.

Hoegerle, S., Ghanem, N., Altehoefer, C., Schipper, J., Brink, I., Moser, E., Neumann, H. P. (2003) 18F-DOPA positron emission tomography for the detection of glomus tumours. Eur. J. Nucl. Med. Mol. Imaging 30, 689-694.

Kafie, F. E., Freischlag, J. A. (2001) Carotid body tumors: the role of preoperative embolization. Ann. Vasc. Surg. 15, 237-242.

Lee, J. H., Barich, F., Karnell, L. H., Robinson, R. A., Zhen, W. K., Gantz, B. J., Hoffman, H. T.; American College of Surgeons Commission on Cancer; American Cancer Socienty (2002) National Cancer Data Base report on malignant paragangliomas of the head and neck. Cancer 94, 730-737.

Litle,V. R., Reilly, L. M., Ramos, T. K. (1996) Preoperative embolisation of carotid body tumors: when is it appropriate? Ann. Vasc. Surg. 10, 464-468.

Marchesi, M., Biffoni, M., Jaus, M. O., Nobili Benedetti, R., Tromba, L., Berni, A., Campana, F. P. (1999) Surgical treatment of paragangliomas of the carotid body and other rare localisations. J. Cardiovasc. Surg. (Torino) 40, 691-694.

Miller, R. B., Boon, M. S., Atkins, J. P., Lowry, L. D. (2000) Vagal paraganglioma: the Jefferson experience. Otolaryngol. Head Neck Surg. 122, 482-487.

Murphy, T. P., Brackmann, D. E. (1989) Effects of preoperative embolization on glomus jugulare tumors. Laryngoscope 99, 1244-1247.

Neumann, H. P., Bausch, B., McWhinney, S. R., Bender, B. U., Gimm, O., Franke, G., Schipper, J., Klisch, J., Altehoefer, C., Zerres, K., Januszewicz, A., Eng, C., Smith, W. M., Munk, R., Manz, T., Glaesker, S., Apel, T. W., Treier, M., Reineke, M., Walz, M. K., Hoang-Vu, C., Brauckhoff, M., Klein-Franke, A., Klose, P., Schmidt, H., Maier-Woelfle, M., Peczkowska, M., Szmigielski, C., Eng, C.; Freiburg-Warsaw-Columbus Pheochromocytoma Study Group (2002) Germ-line mutations in nonsyndromic pheochromocytoma. N. Engl. J. Med. 346, 1459-1466.

Patetsios, P., Gable, D. R., Garrett, W. V., Lamont, J. P., Kuhn, J. A., Shutze, W. P., Kourlis, H., Grimsley, B., Pearl, G. J. Smith, B. L., Talkington, C. M., Thompson, J. E. (2002) Management of carotid body paragangliomas and review of a 30-year experience. Ann. Vasc. Surg. 16, 331-338.

Pellitteri, P., Rinaldo, A., Myssiorek, D., Gary Jackson, C., Bradley, P. J., Devaney, K. O., Shaha, A. R., Netterville, J. L., Manni, J. J., Ferlito, A. (2004) Paragangliomas of the head and neck. Oral Oncol. 40, 563-575.

Persky, M. S., Setton, A., Niimi, Y., Hartman, J., Frank, D., Berenstein, A. (2002) Combined endovascular and surgical treatment of head and neck paragangliomas - a team approach. Head Neck 24, 423-431.

Sanli, A., Oz, K., Ayduran, E., Aydin, S., Altin, G., Eken, M. (2012) Carotid body tumors and our surgical approaches. Indian J. Otolaryngol. Head Neck Surg. 64, 158-161.

Shamblin, W. R., ReMine, W. H., Sheps, S. G., Harrison, E. G. Jr. (1971) Carotid body tumor (chemodectoma). Clinicopathologic analysis of ninety cases. Am.J. Surg. 122, 732-739.

Stoeckli, S. J., Schuknecht, B., Alkadhi, H., Fisch, U. (2002) Evaluation of paragangliomas presenting as a cervical mass on color-coded Doppler sonography. Laryngoscope 112, 143-146. 
270) Prague Medical Report / Vol. 113 (2012) No. 4, p. 262-270

Thabet, M. H., Kotob, H. (2001) Cervical paragangliomas: diagnosis, management and complications. J. Laryngol. Otol. 115, 467-474.

Tikkakoski, T., Luotonen, J., Leinonen, S., Siniluoto, T., Heikkilä, O., Päivänsälo, M., Hyrynkangas, K. (1997)

Preoperative embolization in the management of neck paragangliomas. Laryngoscope 107, 821-826.

Urquhart, A. C., Johnson, J. T., Myers, E. N., Schechter, G. L. (1994) Glomus vagal: paraganglioma of the vagus nerve. Laryngoscope 104, 440-445.

Wang, S. J., Wang, M. B., Barauskas, T. M., Calcaterra, T. C. (2000) Surgical management of carotid body tumors. Otolaryngol. Head Neck Surg. 123, 202-206. 